\title{
Neural and Molecular Features on Charcot-Marie-Tooth Disease Plasticity and Therapy
}

\author{
Paula Juárez ${ }^{1,2}$ and Francesc Palau ${ }^{1,2,3}$ \\ ${ }^{1}$ Unidad de Genética y Medicina Molecular, Instituto de Biomedicina de Valencia, Consejo Superior \\ de Investigaciones Científicas (CSIC), Jaume Roig 11, 46010 Valencia, Spain \\ ${ }^{2}$ CIBER de Enfermedades Raras (CIBERER), ISCIII, 46010 Valencia, Spain \\ ${ }^{3}$ Faculty of Medicine, University of Castilla-La Mancha, 13071 Ciudad Real, Spain \\ Correspondence should be addressed to Francesc Palau, fpalau@ibv.csic.es
}

Received 2 March 2012; Accepted 16 April 2012

Academic Editor: Laurie Doering

Copyright () 2012 P. Juárez and F. Palau. This is an open access article distributed under the Creative Commons Attribution License, which permits unrestricted use, distribution, and reproduction in any medium, provided the original work is properly cited.

In the peripheral nervous system disorders plasticity is related to changes on the axon and Schwann cell biology, and the synaptic formations and connections, which could be also a focus for therapeutic research. Charcot-Marie-Tooth disease (CMT) represents a large group of inherited peripheral neuropathies that involve mainly both motor and sensory nerves and induce muscular atrophy and weakness. Genetic analysis has identified several pathways and molecular mechanisms involving myelin structure and proper nerve myelination, transcriptional regulation, protein turnover, vesicle trafficking, axonal transport and mitochondrial dynamics. These pathogenic mechanisms affect the continuous signaling and dialogue between the Schwann cell and the axon, having as final result the loss of myelin and nerve maintenance; however, some late onset axonal CMT neuropathies are a consequence of Schwann cell specific changes not affecting myelin. Comprehension of molecular pathways involved in Schwann cell-axonal interactions is likely not only to increase the understanding of nerve biology but also to identify the molecular targets and cell pathways to design novel therapeutic approaches for inherited neuropathies but also for most common peripheral neuropathies. These approaches should improve the plasticity of the synaptic connections at the neuromuscular junction and regenerate cell viability based on improving myelin and axon interaction.

\section{Introduction}

Charcot-Marie-Tooth disease (CMT) is a clinical and genetic heterogeneous group of inherited motor and sensory peripheral neuropathies (HMSN) that affect $17-40$ per 100,000 inhabitants $[1,2]$. Mendelian segregation in families may follow either autosomal dominant, autosomal recessive, or $\mathrm{X}$-linked patterns. Autosomal recessive forms are described more frequently in specific populations and geographical areas such as the Mediterranean basin. Molecular genetic studies and positional cloning, and more recently exome sequencing approaches, have unraveled a wide number of genes involved in the etiology of CMT disease [35]. Molecular genetic studies have been very successful for defining the gene nosology and classification of inherited peripheral neuropathies; more than 40 genes have so far been identified to be associated with CMT and related neuropathies, including rare clinical variants (http://www.molgen.ua.ac.be/CMTmutations/). As an immediate consequence, genetic testing has become an important tool in clinical practice of CMT, and patients and families have been beneficiated of a more specific genetic counseling. CMT is caused by mutations in genes that encode proteins with different locations, including compact and noncompact myelin, Schwann cells, and axons, and that are involved in very different functions, which include compaction and maintenance of myelin, transport through myelin, transcription regulation associated with myelination, cell signaling, cytoskeleton formation, axonal transport, mitochondrial dynamics and metabolism, vesicle and endosomal trafficking, and chaperones. Whatever the metabolic or structural defect that primarily affects the myelin or the 
axon, the final common pathway in peripheral neuropathies is represented by an axonal degenerative process that, in most cases, mainly involves the largest and longest fibers [6-8]. Signals from axons determine whether or not a Schwann cell will alter its phenotype and make myelin. Alternatively, Schwann cell abnormalities may induce axonal degeneration with or without demyelination. Progress has been made toward understanding how particular mutations cause disease, but pathogenic mechanisms remain largely unknown.

The PNS is a complex network of myelinated and nonmyelinated nerves of varying diameters. A myelinated nerve fiber consists of a single continuous neuronal process, the axon, surrounded along the outside by serially arranged Schwann cells, which enwrap the associated axon with their cell membrane in a multilayered specialized structure, the myelin sheath. During development, the acquisition of a myelinating phenotype by the Schwann cell appears to be in response to as yet not understood cues from the axons [9]. When the Schwann cell establishes a one-to-one association with an axon at the promyelinating stage of its development, the program of myelination is started and becomes a myelinating Schwann cell. In contrast, Schwann cells that do not establish this relationship with an axon do not activate the program of myelin gene expression and become nonmyelinating Schwann cells [10]. Interestingly, this decision process is directed by the axons, as maintenance of myelin depends on axon and axon integrity. Other example of the influence of the axons on Schwann cells is the establishment of an electrically insulate node of Ranvier. Numerous molecules mediate specific aspects of the interactions between peripheral axons and Schwann cells [11] including MAG, p75, IGF1, integrins, and TGF$\beta$. Neuregulin 1 (Nrg1) and its receptors, the ErbB receptors tyrosine kinases, have emerged as key regulators of axon-Schwann cell interactions at every stage. Spinal cord motoneurons, dorsal root ganglia (DRG) sensory neurons, and autonomic neurons express Nrg1 [12], and Schwann cell express both ErbB2 and ErbB3 receptors [13]. In addition to its previously known roles in proliferation and myelination, Nrg1 type III controls Schwann cell migration. Talbot's group have recently demonstrated that Nrg1 type III is an essential signal that controls Schwann cell migration to ensure that they are present in the correct numbers and positions in developing nerves [14]. Inherited demyelinating neuropathies provide examples of how the axons are also dependent on Schwann cells. The molecular studies on the progressive axonal degeneration seen in demyelinating CMT rodent models have demonstrated that they are likely to be the result of abnormalities in Schwann cell-axonal interactions [15-17].

\section{CMT: Inheritance and Phenotypes of Motor and Sensory Neuropathies}

CMT disease refers to peripheral neuropathies that affect both motor and sensory nerves. They are classically subdivided into "primary demyelinating" forms (CMT disease type 1 or CMT1), which are defined by a characteristic reduction of nerve conduction velocity (NCV) and segmental demyelination and remyelination, and "primary axonal" forms (CMT2) that show preservation or mild reduction of NCVs and loss of axons, namely, those of large diameter $(\geq 8 \mu \mathrm{m})$. As information about CMT2 is increasing, it has become evident that the distinction between CMT1 and CMT2 is less clear-cut than what was originally believed [18]. Diagnosis of CMT2 can be difficult; however, as many authors have noted, this type of CMT appears to have greater variability in its clinical presentation than CMT1. The CMT clinical phenotype is the consequence of a progressive axonal loss and degeneration affecting preferentially the longest axons, whatever the underlying primary pathogenic mechanism, either a myelinopathy and/or axonopathy [6, 7, 19]. CMT is predominantly a large-fibre neuropathy, but as sural nerve biopsies sometimes show, small fibres may be involved as well. In spite of the surprising variability of genes involved in the pathogenesis of CMT, common molecular pathways have been identified within Schwann cells and axons that cause these genetic neuropathies [5]. A review of some of most frequent forms is useful to define relevant clues to the pathogenesis of CMT and to sum up therapeutic interventions oriented to modulate the plasticity of these neuropathies (Figure 1).

\subsection{Autosomal Dominant Demyelinating Neuropathies:} CMT1. Approximately $60 \%$ of CMT patients show a predominantly demyelinating peripheral neuropathy and are classified as CMT1. The main subtype is CMT1A $[20,21]$, accounting for $40-50 \%$ of all CMT cases, which is associated with an autosomal dominant $1.4 \mathrm{Mb}$ duplication on chromosome $17 \mathrm{p} 11.2$ that includes the peripheral myelin protein 22 gene (PMP22) [22, 23], a dosage-sensitive gene, expressed predominantly in the compact myelin of Schwann cells of the PNS. Less commonly, point mutations in PMP22 have been also associated to CMT1. The mirror mutation, that is, the $1.4 \mathrm{Mb}$ deletion $[24,25]$, and more rarely nonsense or frameshift PMP22 mutations [26, 27] cause the myelinopathy called hereditary neuropathy with liability to pressure palsies (HNPPs) [28, 29]. Thus, duplicated or deleted of PMP22 gives rise to demyelinating neuropathies and secondary axonal loss or abnormalities by a mechanism of gene dosage. When overexpressed in cultured cells or in transgenic mice overexpressing the human gene, PMP22 reaches late endosomes and forms protein aggregates that are ubiquitinated [30]. Removal of preexisting aggresomes formed by endogenous PMP22 is aided by autophagy [31]. A second cellular mechanism that can influence protein aggregation is the heat shock response. In proteasome-inhibited cells, overexpressed wild-type and mutant PMP22, as well as the spontaneous aggregates in neuropathic mouse nerves, recruits heat shock proteins [31]. The formation of aggresomes is a protective response of the cell that concentrates misfolded proteins in a central location to activate an autophagic response [32]. Fortun et al. propose a protective role for chaperones in preventing the accumulation of misfolded proteins. Elevated 


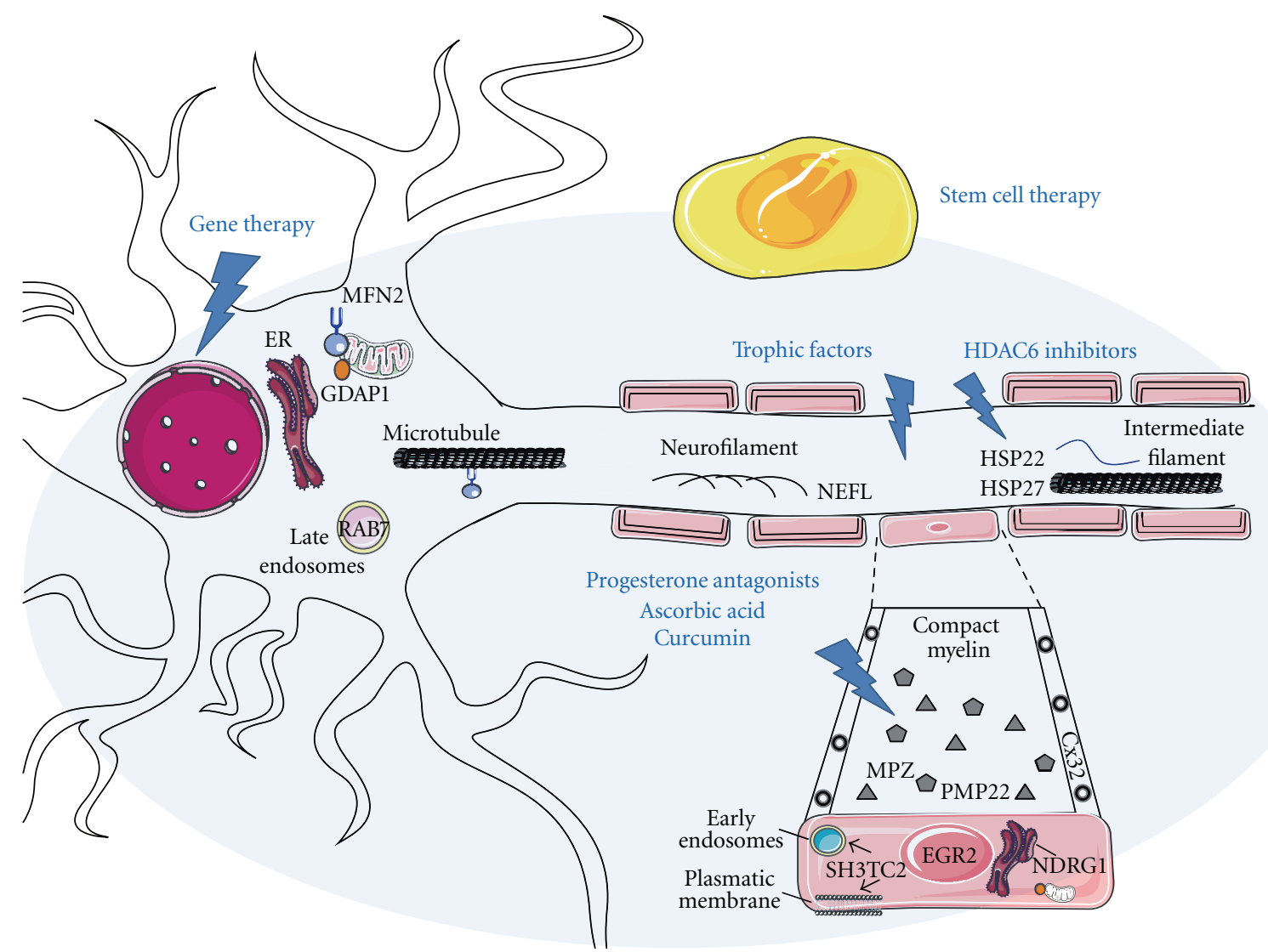

Figure 1: Peripheral nerve structure and cell localization of some CMT proteins at either the Schwann cell and myelin or the neuronal axon. (a) PMP22 and P0 are structural proteins located at the compact myelin and Cx32 at the noncompact myelin in the paranode (and also at the Schmidt-Lanterman incisures). Some other demyelinating CMT-associated molecules are SHT3TC2 at the plasmatic membrane and related to early endosomes and endosome recycling, the transcription factor ERG2 working in early promyelination programme, and NDRG1 that is ubiquitously expressed and has been proposed to play a role in growth arrest and cell differentiation, possibly as a signaling protein shuttling between the cytoplasm and the nucleus. Proteins mainly related to axonal CMT are associated with neurofilaments (NEFL), late endosomes (RAB7), mitochondria, endoplasmic reticulum and microtubules (MFN2 and GDAP1), or intermediate filaments (HSP22 and HSP27). (b) A ray sign indicates the main location where drugs or advanced therapies are acting. Stem cell therapy is represented as an open shadow grasping the neuron soma and axon and the Schwann cell. Trophic factors may be delivered as a drug but also by means of gene vectors or as a part of the local function of therapeutic stem cells. This figure was produced using Servier Medical Art (http://www.servier.com/servier-medical-art/powerpoint-image-bank).

PMP22 expression might perturb Schwann cell function by interfering with the intracellular sorting of PMP22 and other proteins, leading to overloading of the protein degradation machinery. Although demyelination is the pathological and physiological hallmark of CMT1A, the clinical signs and symptoms of this disease, progressive weakness, and sensory loss are produced by axonal degeneration [6]. The characteristic features of the PMP22 mutant Trembler and Trembler-J mice [33], particularly the minimal or abnormal myelination and reduced axonal diameter, have made them very attractive models to define the mechanisms by which defective peripheral myelination can modify axonal properties.

CMT1B is caused by mutations in the major myelin protein zero gene $(M P Z)$, which comprises approximately $50 \%$ of myelin protein, and is necessary for both normal myelin structure and function $[34,35]$. To date there are more than 150 different mutations in MPZ known to cause
CMT1B in patients, which include missense, nonsense, small insertion/deletion, and splice site mutations. Based on clinical studies, Shy and coworkers' [36] patients fall into two distinct phenotype groups: one causing delayed motor development and marked slow nerve conduction and a second one usually associated with late-onset neuropathy, which allows developmental myelination, but eventually leads to axonal degeneration with minimal evidence of demyelination. It is difficult to make genotype-phenotype correlations because mutations in MPZ impair the adhesive function of myelin protein zero (P0), its subcellular trafficking, or both. Either abnormal gain-of-function effects (toxicity of misfolded protein) or reduced amounts of P0 (haploinsufficiency) could, therefore, underlie the clinical phenotype [37]. Pennuto and collaborators demonstrated that the unfolded protein response (UPR) activated by overload of misfolded proteins in the ER was responsible for demyelination in a CMT1B mouse model [38]. A recent 
work has identified that an increased gene dosage of $M P Z$ is directly involved in the pathogenesis of human peripheral nerves [39].

2.2. Autosomal Dominant Axonal Neuropathies: CMT2. CMT2 has a highly heterogeneous genotype. Mutations in the mitofusin 2 gene (MFN2) cause CMT2A and account for about $20 \%$ of CMT 2 cases. Other less frequently mutated genes are MPZ (CMT2J), which also causes CMT1B, and the neurofilament light chain gene (NEFL) [40,41].

CMT2A MFN2 participates in the fusion pathway of the mitochondrial dynamics $[42,43]$ and is also involved in the relationship of mitochondria with endoplasmic reticulum (ER); furthermore, depletion of MFN2 causes a disruption of mitochondrial dynamics and abnormalities in $\mathrm{Ca}^{2+}$ homeostasis [44]. Mechanisms that have been proposed to explain the pathophysiology of CMT2A associated with MFN2 dysfunctions include a defect in mitochondrial fusion, leading to a loss of mtDNA, and impairment in oxidative phosphorylation and cell bioenergetics [45]. Current models propose that a mitochondrial transport defect could be the cause of CMT2A. Zhao et al. were the first to link axonal cargo transport dysfunction to CMT2A [46]. Based on several studies $[47,48]$, it is tempting to speculate that MFN2 could be part of a motor complex involved in anterograde movement of mitochondria. So far, two transgenic mouse models expressing pathogenic mutations have been generating, $M f n 2^{\mathrm{T} 105 \mathrm{M}}$ [49] and $M f n 2^{\mathrm{R} 94 \mathrm{Q}}$ [50]. Loss of MFN2 profoundly and selectively disrupts axonal mitochondrial transport [51], which indicates its integral role in the regulation of mitochondrial transport, and the important implications for understanding the pathophysiology of CMT2A.

To date, up to 18 neurofilament light (NEFL) mutations have been associated with axonal CMT2E [52, 53]. A conditional mouse model, carrying the P22S mutation, mimics many aspects of the human CMT2E disease, including motor disability, abnormal muscle morphology, and denervation events [54]. These results highlight the importance of the integrity of the neurofilament network for neuronal function and suggest that the disease symptoms caused by the NEFL ${ }^{P 22 S}$ mutation might result from axonal transport defects rather than deleterious effects of large neurofilament aggregates.

2.3. X-Linked CMT. With a frequency of about $10 \%$, CMTX1 [55] is the second most common inherited neuropathy. CMTX1 is genetically defined by mutations in the gene GJB1, which encodes the gap junction protein connexin-32 (Cx32) on the Xq13 chromosome [56]. Cx32 is localized in the noncompacted myelin sheath of large diameter fibers, forming the functional channels that allow for the rapid transport of ions and small nutrients between coupled cells [37]. Although Cx32 expression is not limited to the peripheral nervous system (it is also expressed by white matter oligodendrocytes), Cx32 mutations are associated only with CMT [57]. So far, more than 270 mutations that alter the structure of $\mathrm{Cx} 32$ have been reported, and most of these probably cause a partial or complete loss of function. Cx32-deficient mice have prominent adaxonal changes at the ultrastructural level, and a similar pathomechanism is observed in humans [58].

\subsection{Autosomal Recessive CMT: Demyelinating CMT4 and} Axonal AR-CMT2 Variants. In 2002, Baxter et al. [59] and Cuesta et al. [60] demonstrated that mutations in the ganglioside-induced differentiation-associated protein-1 (GDAP1) gene cause autosomal recessive CMT neuropathy. This finding was fascinating, as the two reports differed markedly with respect to the phenotypes of their families. Cuesta et al. described families who had an axonal phenotype (AR-CMT2K), whereas Baxter's families showed a demyelinating phenotype (CMT4A). Both slow and normal NCVs have been reported in patients, and many of the cases show a severe phenotype and have their onset in childhood. However, mild forms segregating as an autosomal dominant phenotype have also been reported [61]. Mutations have been described in every exon and include missense, nonsense, splicing site, short deletions, and insertion mutations. GDAP1 belongs to a glutathione S-transferase enzyme subfamily [62] that is mainly expressed in neurons $[63,64]$ but also in Schwann cells [65]. GDAP1 is a mitochondrial protein [63] located in the mitochondrial outer membrane $(\mathrm{MOM})$ acting as a regulator of mitochondrial dynamics $[65$, 66]. The effect of GDAP1 mutations in mitodynamics seems to depend on the inheritance pattern [67]. Overexpression of GDAP1 in COS7 or HeLa cells causes mitochondrial fragmentation or fission and a substantial accumulation of mitochondria around the nucleus. Rescue experiments in Saccharomyces cerevisiae defective mutans for fission and fusion genes have shown that GDAP1 rescues the phenotype of the fission-associated gene Fis1. In particular, the recovery of G2/M delay suggests that both Fis1p and GDAP1 may affect the interaction of mitochondria with microtubules [68], an aspect that may relate GDAP1 to mitochondrial transport or movement in axons.

CMT4C neuropathy, which is caused by mutations in the SH3TC2 gene, is the most common cause of the autosomal recessive form of demyelinating CMT. SH3TC2 is specifically expressed in Schwann cells and is necessary for proper myelination of peripheral axons. Analysis of the murine model of CMT4C revealed that the capacity of SH3TC2deficient Schwann cells to properly myelinate underlying axons is affected at the early stages of myelination, which is in line with the early onset of the neuropathy reported in CMT4C patients [69-71]. However, its exact role in myelin biology remains to be determined. Recent data demonstrated that SH3TC2 localizes at the plasma membrane and in endocytic vesicles [72-74] and that it interacts with the small GTPase Rab11, which is known to regulate the recycling of internalized membranes and receptors back to the plasma membrane. Further protein binding studies and transferrin receptor trafficking assays revealed that SH3TC2 together with Rab11 indeed affect the dynamics of endocytic recycling [75]. 


\section{Cellular and Molecular Bases of Nerve Regeneration and Plasticity in CMT Neuropathies}

The discovery of many genes involved in CMT disease has provided a unique opportunity to understand the critical molecular pathways involved in peripheral axon stability and length-dependent peripheral nerve disease [17, 18]. An important concept in peripheral neuropathy is that many types are characteristically length dependent; that is, the longest axons in the body are affected first and most profoundly. The length-dependent distribution supports the concept that the major site of pathology is in the axon itself, rather than the cell body. Furthermore, it suggests that shorter axons are either less susceptible or better able to compensate for certain insults that are longer axons, leading to the degeneration of the distal regions of the longest axons first [76]. Peripheral neuropathies presenting a distal nonterminal axonopathy represent the most common nerve diseases. Their long-term outcome depends on the balance of two processes: the degree or rate of axonal degeneration and the ability of the nascent axon tips to regenerate efficiently. One strategy to alter these processes would be to improve the efficiency of regeneration by using trophic factors such as neurotrophins [77], moving them from benchto bedside. Prolonged denervation could lead to decreased regeneration capacity because of reduction in the expression of regeneration-associated Schwann cell molecules, such as neurotrophic factors and receptors. Therefore, Schwann cells might remain in a growth-supportive mode for prolonged periods or they have to be transformed into a competent premyelinating state to initiate and complete myelination. The functional significance of regeneration is to allow reinnervation of target organs and restitution of their corresponding functions. The materials for axonal growth are mainly provided by the cell body via axonal transport $[78,79]$, but more recently the contribution of local axonal synthesis and degradation of proteins has been identified $[80,81]$. Increased energy demands on the neuron to propagate action potentials, and decreased trophic factor support from denervated Schwann cells or muscle are other potential mechanisms that may also contribute to axonal degeneration in demyelinating neuropathies (reviewed in [16]). An unanswered question with respect to all CMT1 forms is why mutant Schwann cells fail to support axonal function and survival [37]. An important direction is the development of therapeutic strategies that enhance axonal regeneration and promote selective target reinnervation; in addition, modulation of the central nervous system reorganization to improve functional recovery but also diminishing undesirable consequences has been proposed as well [82].

\section{CMT Pharmacological and Biological Therapies}

CMT disease course and severity vary according to CMT type, causative gene, and mutation change, but considerable phenotypic variability may occur also within the same CMT type. Understanding the molecular pathogenesis of inherited neuropathies is essential for the development of rational therapies (Figure 1). While much remains to be learned, it is clear that most are caused by the expression of a mutant allele(s) in myelinating Schwann cells or neurons. For recessive neuropathies, in principle it is possible to "replace" the defective gene by introducing a normal version. For dominant neuropathies, the situation is even more complex, as these are likely to be caused by a toxic gain of function that is not necessarily related to the normal function of the gene product. Nevertheless, for dominant demyelinating neuropathies caused by altered gene dosage, reestablishing the normal level of gene expression might be of benefit. The pathogenic mechanism in CMT1A duplication is attributed to an excess gene copy number of PMP22, leading to protein overexpression [28], and factors that modify the expression levels of PMP22 might potentially be effective for treatment. A proof of concept that demyelination can be reversed by normalizing expression of PMP22 was provided through a transgenic mouse model [83]. Research is focused on developing new treatment strategies to target the regulation of PMP22 gene dosage. Two compounds that have been shown to alter PMP22 mRNA levels in rodents are ascorbic acid and progesterone and progesterone antagonists.

Ascorbic acid reduced the severity of neuropathy in transgenic mice overexpressing PMP22, an animal model of human CMT1A, compared with untreated mice [84]. Ascorbic acid promotes myelination in vitro and decreases PMP22 mRNA levels through a cAMP-mediated pathway $[84,85]$. Evidence of efficacy of ascorbic acid in the animal model prompted initiation of randomized controlled trials to test the efficacy of ascorbic acid in patients with CMT1A. Results from a phase 3, multicentre, placebo-controlled, double-blind randomized trial to assess the efficacy and tolerability of chronic ascorbic acid treatment in patients with CMT1A in Italy and the UK have been just published [86]. Unfortunately, ascorbic acid supplementation had no significant effect on neuropathy compared to placebo after 2 years followup, suggesting that no evidence is available to support treatment with ascorbic acid in adults with CMT1A.

It is known that progesterone and derivates are able to increase $M P Z$ and $P M P 22$ gene expression in vitro [87]. In order to test if progesterone can modulate the progressive neuropathy caused by moderate overexpression of PMP22, Sereda and collaborators [88] administrated daily subcutaneous injections of progesterone and the progesterone antagonist, onapristone, to a transgenic rat model of CMT1A during 7 weeks and showed that onapristone reduced PMP 22 mRNA by $15 \%$, resulting in clinical and neuropathological improvement. A long-term study reaffirmed these results and shed light to the axonal degeneration process seen in CMT1A patients, by reducing progressive muscle atrophy and preventing axonal loss without altering myelin sheath thickness [89]. Unfortunately, onapristone and currently available progesterone antagonists are too toxic to be safely administered to CMT1A patients, so further research is ongoing to develop suitable compounds for future clinical trials. To accomplish this goal, the Charcot-Marie-Tooth Association (CMTA) has established 
the Strategy to Accelerate Research (STAR) to specifically fund CMT-related research (http://www.cmtausa.org/). High-throughput screens are trying to identify compounds that are already FDA approved, so it could accelerate the drug development process and start phase III clinical trials in $3-5$ years.

Another interesting and promising molecule is curcumin, which plays a role stimulating the translocation of misfolded proteins from the endoplasmic reticulum to the plasma membrane, thereby reducing cytotoxicity of the mutant proteins. This mechanism might be helpful for selected CMT1A and CMT1B forms, in which various $P M P 22$ and MPZ mutations are known to cause intracellular accumulation of mutant proteins. Oral curcumin mitigates the clinical and neuropathologic phenotype of Trembler-J mouse model of CMT1A, inhibiting Schwann cell apoptosis and increasing axonal caliber and myelin thickness. Furthermore, this positive clinical response to curcumin occurs in a dose-dependent manner and is reversed after withdrawal of treatment without side effects. Recent cell-based studies showed that mutant P0 could accumulate in the ER and induce apoptosis. This aggregation-induced apoptosis was abrogated by pretreatment with curcumin [90]. These findings suggest a potential therapeutic role of curcumin in selected forms of inherited peripheral neuropathies. There is also evidence that $M P Z$ mutations with ER-retention of the mutated protein cause UPR activation rather than apoptosis [38] so this mechanism could also be relevant as a therapeutic target.

Regarding axonal CMT, in a transgenic mouse model for mutant HSBP1-induced CMT2 and distal HMN type 2B [91], mutant HSBP1, also known as HSP27, leads to severe axonal transport defects induced by a decrease in acetylated tubulin abundance in peripheral nerves. The phenotype was partially restored and the axonal transport defects were rescued when mice were treated with HDAC6 inhibitors. Histone deacetylase 6 (HDAC6) is the major enzyme with $\alpha$-tubulin deacetylating activity. For this study the authors used a nonspecific inhibitor, trichostatin A (TSA), and two highly selective inhibitors: tubacin and tubastatin A, which resulted to be more effective compared to TSA when axonal transport and CMT phenotype were assessed. As some HDAC6 inhibitors have entered into clinical trials for cancer treatment and other neurological disorders such as Friedreich's ataxia [92], it is rationale to think that the same drug approach could be tested in CMT patients.

Schwann cell pathology damages the delicate myelinaxon interaction and can lead to axonal degeneration [6], but pronounced axonal pathology has also been observed even in genetic models in which axons are associated with normal appearing myelin sheaths. Thus, a therapeutic approach focusing on preventing this intimate connection could be providing trophic factor support to degenerating axons, which may be useful for a number of CMT neuropathies, either primary myelinopathies or primary axonopathies. The main conceptual problem with this approach is the diversity of trophic factors and PNS neurons, especially because different kinds of neurons respond to different trophic factors [16]. Only neurons with the proper receptors typically respond to a given growth factor. Receptor-mediated, retrograde axonal transport delivers the trophic factor to neuronal cell bodies, where they act. Axonal elongation requires an adequate substrate of trophins and tropic factors, provided by reactive Schwann cells and the extracellular matrix within the degenerated nerve [93]. Another potentially approach involves the manipulation of Schwann cellaxonal signal transduction pathways. Neuregulin-1 enhances axonal regeneration [94] by acting on Schwann cells as PNS neurons do not express neuregulin receptors. Axons express neuregulin-1 type III (Nrg1-III) on their surface, which binds to ErbB receptors on Schwann cells as part of a process that initiates myelination. Therefore, Nrg1-III acts as a juxtacrine signal. Nrg1-III binds to ErbB3 and promotes ErbB2 phosphorylation of tyrosine residues in the cytoplasmic domain of both ErbB2 and ErbB3 receptors [95]. Activation of the ErbB receptors leads to signaling through multiple signaling cascades including PI3K/Akt, Erk1/2, JNK, and FAK [96]. Three families of trophic factors are particularly important for PNS neurons: the neurotrophin family, the glial-derived neurotrophic factor (GDNF), and the ciliary neurotrophic factor (CNTF) family of cytokines. Sahenk and collaborators studied the ability of mutant Schwann cells to respond to exogenous neurotrophin-3 (NT3 ) in two CMT1A animal models [97]. NT-3-treated animals presented improved nerve regeneration and the associated myelination process. Furthermore, at the early stages of regeneration-associated myelination, NT-3 stabilizes the axonal cytoskeleton locally by inducing neurofilament phosphorylation when axon sprouts become enwrapped by Schwann cells [77]. In contrast, BDNF, which belongs to the neurotrophin family but is not part of the Schwann cell survival loop [98], showed no effect upon axonal growth or cytoskeletal neurofilament pathology. Unfortunately, despite the promising results in animal studies, no studies have been successful in humans, probably due to a poor delivery and short half-lives of the trophic and growth factors. Targeting the correct combination of trophic factors to neurons or Schwann cells at the optimal time may be necessary to achieve meaningful results. A combination of trophic factors or engineered "pan-neurotrophic factors" [99] might be more beneficial than a single factor.

The short half-life of most neurotrophic factors would require either multiple administrations or a continuous infusion of the therapeutic molecules in order to achieve an adequate and effective local concentration. Knowing the molecular basis of inherited diseases prompts immediate consideration of gene therapy. Gene therapy can be defined as a strategy to transfer biologically relevant genetic material (usually mutant genes or genes delivering trophic and growth factors) into affected cells in the body to treat disease. For instance, delivering neurotrophic factors to the healing nerve ends, favoring survival and regeneration of both sensory and motor axons, could, ultimately, allow the recovery of nervous functions [100]. Viral vectors and plasmid DNA have been widely used for treating human disease models and patients. Expression of the gene might be modulated by the introduction of regulatory elements for the controlled or 
tissue-specific expression of the desired molecule. Until now, three major classes of viral vectors, based on adenovirus, adeno-associated virus (AAV), and herpes simplex virus (HSV), have been exploited to target the PNS. Although not originally neurotropic, adenoviral and AAV vectors are able to transduce spinal sensory and motor neurons after either intramuscular or intraneural injections. When injected at the site of a nerve injury, these vectors are retrogradely transported to motor neuron cell bodies and can thus be exploited to deliver therapeutic genes along the route of the nerve. However, the strong absorption of both adenoviral and AAV vectors to skeletal muscle fibers might represent a limitation for efficient neuronal transduction and retrograde transport [101]. A recent study in mice has characterized the tropism and transduction efficiency of different AAV pseudotypes after sciatic nerve injection. Among the pseudotypes tested, AAV2/1 transduced both Schwann cells and neurons, AAV2/2 infected only sensory neurons, and AAV2/8 preferentially transduced Schwann cells, proving the utility of AAVs as gene therapy vectors [102]. A few approaches have used plasmids as vehicles to deliver therapeutic genes to peripheral nerves. In these cases, the skeletal muscle has been the preferred site for delivery and expression of the transgene. For instance, intramuscular delivery of a plasmid encoding for vascular endothelial growth factor (VEGF) showed a protective role against myelin wasting and axonal loss [103]. Viral vectors have been modified so that they are unable to cause disease. Unfortunately, they have caused immunologic reactions, which currently limit their use. In contrast, plasmid DNA is nonimmunogenic, but it is characterized by poor delivery efficiency, and proteins made from it have only been produced in target organs for a short time. Progress in this area demands more sophisticated delivery systems and more knowledge of the molecular pathogenesis of neuropathies.

Schwann cells, and their basal lamina, represent the key component of nerve regeneration, as they serve as scaffolds for the regenerating axons, which grow through the empty basal lamina tubes. Schwann cells, however, have limited clinical applications since the culture of an adequate quantity of cells to achieve optimal conditions for transplantation in nerve conduits is time consuming and requires particular care for in vitro expansion and a constant input of growth factors. Moreover, Schwann cells are not easily accessible without nerve biopsy and bear the need to sacrifice an autologous nerve, with the related complications. Due to all these difficulties, the field of stem cell therapy for peripheral neuropathies has been explored. Adult stem cells show ability to differentiate into neuroprogenitor-type cells [104, 105]. Stem cells could be differentiating into neurons, which will generate new axons to contact their targets or Schwann cells enwrapping demyelinating axons and secreting trophic factors. Bonemarrow-derived mesenchymal stem cells (MSCs) can be induced to differentiate into Schwann cells [106], improving myelin formation and nerve regeneration in vivo after their transplantation into different models of peripheral nerve injury $[107,108]$. Adipose tissue has been also indicated as a novel and promising source of multipotent cells (adiposederived stem cells, ASCs), which can be differentiated into a neuronal phenotype $[109,110]$, and in terms of clinical use, they may be harvested by conventional liposuction procedure under local anaesthesia. The frequency of stem cells in adipose tissue is 100- to 1000-fold higher than that in bone marrow, which is a considerable advantage as it reduces the period of expansion of the stem cells prior to differentiation. Terenghi's group showed how ASCs could be differentiated towards a Schwann cell-like phenotype, expressing markers like S-100, glial fibrillary acidic protein (GFAP) and P75 neurotrophin receptor and enhancing neurite outgrowth in an in vitro co-culture model [111]. More recently, expression of myelin proteins P0 and PMP22 after differentiation of both ASC and MSC [112] and the neurotrophic potential shown in vitro from differentiated adipose-derived stem cells (dASCs) with a brief term in vivo study have been described [113].

\section{Conclusions}

Charcot-Marie-Tooth disease is a generalized disorder of motor and sensory peripheral nerves. Three major points deserve attention: (1) disease pathophysiology of both myelinopathies and axonopathies forms are the consequence of altered Schwann cell-axon communication; abnormal intercellular contact and signaling induce neurodegeneration and axonal loss, which ultimate produce muscular atrophy and weakness, (2) the primary cause is the genetic mutation in any of the more than 40 genes causing CMT or related neuropathies, and (3) a major therapeutic target is the regeneration of cell viability based on improving myelin and axon interaction. Therapies promoting plasticity changes in axons and Schwann cells require not only new therapeutic drug, gene, or cell approaches but also proper delivery systems targeted to the pathological cellular structures.

\section{Conflict of Interests}

Both P. Juárez and F. Palau have no conflict of interest.

\section{Acknowledgments}

The research work in our laboratory is funded by grants from the Spanish Ministry of Science and Innovation, the Instituto de Salud Carlos III (ISCIII-IRDiRC programme), the Generalitat Valenciana (Prometeo programme), the European Commission FP7, the Fundació Marató TV3, and the Fundación Isabel Gemio. CIBERER is an initiative in cooperative research on rare diseases from the Instituto de Salud Carlos III.

\section{References}

[1] H. Skre, "Genetic and clinical aspects of Charcot Marie Tooth's disease," Clinical Genetics, vol. 6, no. 2, pp. 98-118, 1974.

[2] O. Combarros, J. Calleja, J. M. Polo, and J. Berciano, "Prevalence of hereditary motor and sensory neuropathy in 
Cantabria," Acta Neurologica Scandinavica, vol. 75, no. 1, pp. 9-12, 1987.

[3] J. R. Lupski and C. A. Garcia, "Charcot-Marie-Tooth peripheral neuropathies and related disorders," in The Metabolic and Molecular Bases of Inherited Disease, C. R. Scriver, A. L. Beaudet, W. S. Sly et al., Eds., pp. 5759-5788, McGraw-Hill, New York, NY, USA, 8th edition, 2000.

[4] K. Szigeti and J. R. Lupski, "Hereditary motor and sensory neuropathies," in Emory and Rimoin's Principles and Practice of Medical Genetics, R. L. Rimoin, J. M. Connor, R. E. Pyeritz, and B. R. Korf, Eds., pp. 2946-2962, Churchill Livingstone Elsevier, Philadelphia, Pa, USA, 5th edition, 2007.

[5] A. Patzkó and M. E. Shy, "Update on Charcot-Marie-Tooth disease," Current Neurology and Neuroscience Reports, vol. 11, no. 1, pp. 78-88, 2011.

[6] K. M. Krajewski, R. A. Lewis, D. R. Fuerst et al., "Neurological dysfunction and axonal degeneration in Charcot-MarieTooth disease type 1A," Brain, vol. 123, no. 7, pp. 1516-1527, 2000.

[7] D. Pareyson, V. Scaioli, and M. Laurà, "Clinical and electrophysiological aspects of charcot-marie-tooth disease," NeuroMolecular Medicine, vol. 8, no. 1-2, pp. 3-22, 2006.

[8] S. S. Scherer and L. Wrabetz, "Molecular mechanisms of inherited demyelinating neuropathies," Glia, vol. 56, no. 14, pp. 1578-1589, 2008.

[9] R. Mirsky and K. R. Jessen, "The neurobiology of Schwann cells," Brain Pathology, vol. 9, no. 2, pp. 293-311, 1999.

[10] H. Webster and F. De, "Development of peripheral nerve fibres," in Peripheral Neuropathy, P. J. Dyck, P. K. Thomas, J. W. Griffin, P. A. Low, and J. F. Poduslo, Eds., pp. 243-266, WB Saunders, Philadelphia, Pa, USA, 3th edition, 1993.

[11] G. Corfas, M. O. Velardez, C. P. Ko, N. Ratner, and E. Peles, "Mechanisms and roles of axon-Schwann cell interactions," Journal of Neuroscience, vol. 24, no. 42, pp. 9250-9260, 2004.

[12] G. Corfas, K. M. Rosen, H. Aratake, R. Krauss, and G. D. Fischbach, "Differential expression of ARIA isoforms in the rat brain," Neuron, vol. 14, no. 1, pp. 103-115, 1995.

[13] D. Meyer and C. Birchmeier, "Multiple essential functions of neuregulin in development," Nature, vol. 378, no. 6555, pp. 386-390, 1995.

[14] J. R. Perlin, M. E. Lush, W. Z. Stephens, T. Piotrowski, and W. S. Talbot, "Neuronal Neuregulin 1 type III directs Schwann cell migration," Development, vol. 138, pp. 4639-4648, 2011.

[15] S. De Waegh and S. T. Brady, "Altered slow axonal transport and regeneration in a myelin-deficient mutant mouse: the trembler as an in vivo model for schwann cell-axon interactions," Journal of Neuroscience, vol. 10, no. 6, pp. 1855-1865, 1990.

[16] C. Massicote and S. S. Scherer, "Neuropathies-translating causes into treatments," in Neuroscience, Molecular Medicine, and the Therapeutic Transformation of Neurology, S. G. Waxman, Ed., pp. 401-414, Elsevier Science, London, UK, 2004.

[17] M. E. Shy, "Biology of peripheral inherited neuropathies: schwann cell axonal interactions," Advances in Experimental Medicine and Biology, vol. 652, pp. 171-181, 2009.

[18] S. Züchner and J. M. Vance, "Mechanisms of disease: a molecular genetic update on hereditary axonal neuropathies," Nature Clinical Practice Neurology, vol. 2, no. 1, pp. 45-53, 2006.

[19] T. Sevilla, T. Jaijo, D. Nauffal et al., "Vocal cord paresis and diaphragmatic dysfunction are severe and frequent symptoms of GDAP1-associated neuropathy," Brain, vol. 131, no. 11, pp. 3051-3061, 2008.

[20] D. Pareyson and C. Marchesi, "Diagnosis, natural history, and management of Charcot-Marie-Tooth disease," The Lancet Neurology, vol. 8, no. 7, pp. 654-667, 2009.

[21] J. Berciano, A. García, E. Gallardo, C. Ramón, and O. Combarros, "Phenotype and clinical evolution of charcot-marietooth disease type 1A duplication," Advances in Experimental Medicine and Biology, vol. 652, pp. 183-200, 2009.

[22] J. R. Lupski, R. M. de Oca-Luna, S. Slaugenhaupt et al., "DNA duplication associated with Charcot-Marie-Tooth disease type 1A," Cell, vol. 66, no. 2, pp. 219-232, 1991.

[23] P. Raeymaekers, V. Timmerman, E. Nelis et al., "Duplication in chromosome 17p11.2 in Charcot-Marie-Tooth neuropathy type 1a (CMT 1a)," Neuromuscular Disorders, vol. 1, no. 2, pp. 93-97, 1991.

[24] P. F. Chance, M. K. Alderson, K. A. Leppig et al., "DNA deletion associated with hereditary neuropathy with liability to pressure palsies," Cell, vol. 72, no. 1, pp. 143-151, 1993.

[25] P. F. Chance, N. Abbas, M. W. Lensch et al., "Two autosomal dominant neuropathies result from reciprocal DNA duplication/deletion of a region on chromosome 17," Human Molecular Genetics, vol. 3, no. 2, pp. 223-228, 1994.

[26] G. A. Nicholson, L. J. Valentijn, A. K. Cherryson et al., "A frame shift mutation in the PMP22 gene in hereditary neuropathy with liability to pressure palsies," Nature Genetics, vol. 7, no. 1, p. 113, 1994.

[27] S. Bort, E. Nelis, V. Timmerman et al., "Mutational analysis of the MPZ, PMP22 and Cx32 genes in patients of Spanish ancestry with Charcot-Marie-Tooth disease and hereditary neuropathy with liability to pressure palsies," Human Genetics, vol. 99, no. 6, pp. 746-754, 1997.

[28] J. R. Lupski and P. F. Chance, "Hereditary motor and sensory neuropathies involving altered dosage or mutation of PMP22: the CMT1A duplication and HNPP deletion," in Peripheral Neuropathy, P. L. Dyck and P. K. Thomas, Eds., pp. 1659-1680, Elsevier Saunders, Philadelphia, Pa, USA, 4th edition, 2005.

[29] J. Infante, A. García, O. Combarros et al., "Diagnostic strategy for familial and sporadic cases of neuropathy associated with 17p11.2 deletion," Muscle and Nerve, vol. 24, no. 9, pp. 1149-1155, 2001.

[30] J. Fortun, J. C. Go, J. Li, S. A. Amici, W. A. Dunn Jr., and L. Notterpek, "Alterations in degradative pathways and protein aggregation in a neuropathy model based on PMP22 overexpression," Neurobiology of Disease, vol. 22, no. 1, pp. 153-164, 2006.

[31] J. Fortun, W. A. Dunn Jr., S. Joy, J. Li, and L. Notterpek, "Emerging Role for Autophagy in the Removal of Aggresomes in Schwann Cells," Journal of Neuroscience, vol. 23, no. 33, pp. 10672-10680, 2003.

[32] J. Fortun, J. D. Verrier, J. C. Go, I. Madorsky, W. A. Dunn Jr., and L. Notterpek, "The formation of peripheral myelin protein 22 aggregates is hindered by the enhancement of autophagy and expression of cytoplasmic chaperones," Neurobiology of Disease, vol. 25, no. 2, pp. 252-265, 2007.

[33] K. Aldkofer, R. Naef, and U. Suter, "Analysis of compound heterozygous mice reveals that the Trembler mutation can behave as a gain-of-function allele," Journal of Neuroscience Research, vol. 49, pp. 671-680, 1997.

[34] E. H. Eylar, K. Uyemura, S. W. Brostoff et al., "Proposed nomenclature for PNS myelin proteins," Neurochemical Research, vol. 4, no. 2, pp. 289-293, 1979. 
[35] S. Greenfield, S. Brostoff, E. H. Eylar, and P. Morell, "Protein composition of myelin of the peripheral nervous system," Journal of Neurochemistry, vol. 20, no. 4, pp. 1207-1216, 1973.

[36] M. E. Shy, A. Jáni, K. Krajewski et al., "Phenotypic clustering in MPZ mutations," Brain, vol. 127, no. 2, pp. 371-384, 2004.

[37] K. A. Nave, M. W. Sereda, and H. Ehrenreich, "Mechanisms of disease: inherited demyelinating neuropathies-From basic to clinical research," Nature Clinical Practice Neurology, vol. 3, no. 8, pp. 453-464, 2007.

[38] M. Pennuto, E. Tinelli, M. Malaguti et al., "Ablation of the UPR-Mediator CHOP Restores Motor Function and Reduces Demyelination in Charcot-Marie-Tooth 1B Mice," Neuron, vol. 57, no. 3, pp. 393-405, 2008.

[39] M. H. Maeda, J. Mitsui, B. W. Soong, Y. Takahashi et al., "Increased gene dosage of myelin protein zero causes Charcot-Marie-Tooth disease," Annals of Neurology, vol. 71, pp. 84-92, 2012.

[40] D. Pareyson, "Axonal Charcot-Marie-Tooth disease: the fog is only slowly lifting," Neurology, vol. 68, no. 20, pp. 1649-1650, 2007.

[41] K. Verhoeven, K. G. Claeys, S. Züchner et al., "MFN2 mutation distribution and genotype/phenotype correlation in Charcot-Marie-Tooth type 2," Brain, vol. 129, no. 8, pp. 2093-2102, 2006.

[42] D. C. Chan, "Dissecting mitochondrial fusion," Developmental Cell, vol. 11, no. 5, pp. 592-594, 2006.

[43] K. Okamoto and J. M. Shaw, "Mitochondrial morphology and dynamics in yeast and multicellular eukaryotes," Annual Review of Genetics, vol. 39, pp. 503-536, 2005.

[44] O. M. De Brito and L. Scorrano, "Mitofusin 2 tethers endoplasmic reticulum to mitochondria," Nature, vol. 456, no. 7222, pp. 605-610, 2008.

[45] D. Bach, S. Pich, F. X. Soriano et al., "Mitofusin-2 determines mitochondrial network architecture and mitochondrial metabolism: a novel regulatory mechanism altered in obesity," Journal of Biological Chemistry, vol. 278, no. 19, pp. 17190-17197, 2003.

[46] C. Zhao, J. Takita, Y. Tanaka et al., "Charcot-Marie-Tooth disease type $2 \mathrm{~A}$ caused by mutation in a microtubule motor KIF1B $\beta$," Cell, vol. 105, no. 5, pp. 587-597, 2001.

[47] H. Chen, J. M. McCaffery, and D. C. Chan, "Mitochondrial fusion protects against neurodegeneration in the cerebellum," Cell, vol. 130, no. 3, pp. 548-562, 2007.

[48] S. A. Detmer and D. C. Chan, "Functions and dysfunctions of mitochondrial dynamics," Nature Reviews Molecular Cell Biology, vol. 8, no. 11, pp. 870-879, 2007.

[49] S. A. Detmer, C. V. Velde, D. W. Cleveland, and D. C. Chan, "Hindlimb gait defects due to motor axon loss and reduced distal muscles in a transgenic mouse model of CharcotMarie-Tooth type 2A," Human Molecular Genetics, vol. 17, no. 3, pp. 367-375, 2008.

[50] R. Cartoni, E. Arnaud, J. J. Médard et al., "Expression of mitofusin 2R94Q in a transgenic mouse leads to CharcotMarie-Tooth neuropathy type 2A," Brain, vol. 133, no. 5, pp. 1460-1469, 2010.

[51] A. Misko, S. Jiang, I. Wegorzewska, J. Milbrandt, and R. H. Baloh, "Mitofusin 2 is necessary for transport of axonal mitochondria and interacts with the Miro/Milton complex," Journal of Neuroscience, vol. 30, no. 12, pp. 4232-4240, 2010.

[52] J. R. Lupski, "Axonal Charcot-Marie-Tooth disease and the neurofilament light gene (NF- L)," American Journal of Human Genetics, vol. 67, no. 1, pp. 8-10, 2000.
[53] B. J. Gentil, S. Minotti, M. Beange, R. H. Baloh, J. P. Julien, and H. D. Durham, "Normal role of the low-molecularweight neurofilament protein in mitochondrial dynamics and disruption in Charcot-Marie-Tooth disease," The FASEB Journal, vol. 26, no. 3, pp. 1194-1203, 2011.

[54] F. Dequen, M. Filali, R. C. Larivière, R. Perrot, S. I. Hisanaga, and J. P. Julien, "Reversal of neuropathy phenotypes in conditional mouse model of Charcot-Marie-Tooth disease type 2E," Human Molecular Genetics, vol. 19, no. 13, pp. 2616-2629, 2010.

[55] A. F. Hahn, W. F. Brown, W. J. Koopman, and T. E. Feasby, "Xlinked dominant hereditary motor and sensory neuropathy," Brain, vol. 113, no. 5, pp. 1511-1525, 1990.

[56] J. Bergoffen, S. S. Scherer, S. Wang et al., "Connexin mutations in X-linked Charcot-Marie-Tooth disease," Science, vol. 262, no. 5142, pp. 2039-2042, 1993.

[57] A. Vital, X. Ferrer, A. Lagueny et al., "Histopathological features of X-linked Charcot-Marie-Tooth disease in 8 patients from 6 families with different connexin 32 mutations," Journal of the Peripheral Nervous System, vol. 6, no. 2, pp. 79-84, 2001.

[58] P. Anzini, D. H.-H. Neuberg, M. Schachner et al., "Structural abnormalities and deficient maintenance of peripheral nerve myelin in mice lacking the gap junction protein connexin 32," Journal of Neuroscience, vol. 17, no. 12, pp. 4545-4551, 1997.

[59] R. V. Baxter, K. B. Othmane, J. M. Rochelle et al., "Ganglioside-induced differentiation-associated protein-1 is mutant in Charcot-Marie-Tooth disease type 4A/8q21," Nature Genetics, vol. 30, no. 1, pp. 21-22, 2002.

[60] A. Cuesta, L. Pedrola, T. Sevilla et al., "The gene encoding ganglioside-induced differentiation-associated protein 1 is mutated in axonal Charcot-Marie-Tooth type 4A disease," Nature Genetics, vol. 30, no. 1, pp. 22-25, 2002.

[61] R. Claramunt, L. Pedrola, T. Sevilla et al., "Genetics of Charcot-Marie-Tooth disease type 4A: mutations, inheritance, phenotypic variability, and founder effect," Journal of Medical Genetics, vol. 42, no. 4, pp. 358-365, 2005.

[62] A. Marco, A. Cuesta, L. Pedrola, F. Palau, and I. Marín, "Evolutionary and structural analyses of GDAP1, involved in Charcot-Marie-Tooth disease, characterize a novel class of glutathione transferase-related genes," Molecular Biology and Evolution, vol. 21, no. 1, pp. 176-187, 2004.

[63] L. Pedrola, A. Espert, X. Wu, R. Claramunt, M. E. Shy, and F. Palau, "GDAP1, the protein causing Charcot-Marie-Tooth disease type 4A, is expressed in neurons and is associated with mitochondria," Human Molecular Genetics, vol. 14, no. 8, pp. 1087-1094, 2005.

[64] L. Pedrola, A. Espert, T. Valdés-Sánchez et al., "Cell expression of GDAP1 in the nervous system and pathogenesis of Charcot-Marie-Tooth type 4A disease," Journal of Cellular and Molecular Medicine, vol. 12, no. 2, pp. 679-689, 2008.

[65] A. Niemann, M. Ruegg, V. La Padula, A. Schenone, and U. Suter, "Ganglioside-induced differentiation associated protein 1 is a regulator of the mitochondrial network: new implications for Charcot-Marie-Tooth disease," Journal of Cell Biology, vol. 170, no. 7, pp. 1067-1078, 2005.

[66] F. Palau, A. Estela, D. Pla-Martín, and M. Sánchez-Piris, “The role of mitochondrial network dynamics in the pathogenesis of charcot-marie-tooth disease," Advances in Experimental Medicine and Biology, vol. 652, pp. 129-137, 2009.

[67] A. Niemann, K. M. Wagner, M. Ruegg, and U. Suter, "GDAP1 mutations differ in their effects on mitochondrial dynamics 
and apoptosis depending on the mode of inheritance," Neurobiology of Disease, vol. 36, no. 3, pp. 509-520, 2009.

[68] A. Estela, D. Pla-Martín, M. Sánchez-Piris, H. Sesaki, and F. Palau, "The Charcot-Marie-Tooth related gene GDAP1 complements cell cycle delay at G2/M in S. cerevisiae fis1 defective cells," Journal of Biological Chemistry, vol. 286, pp. 36777-36786, 2011.

[69] L. Kalaydjieva, H. Lochmüller, I. Tournev et al., "125th ENMC International Workshop: Neuromuscular disorders in the Roma (Gypsy) population, p. 23, Naarden, The Netherlands, April 2004," Neuromuscular Disorders, vol. 15, no. 1, pp. 65-71, 2005.

[70] J. Colomer, R. Gooding, D. Angelicheva et al., "Clinical spectrum of CMT4C disease in patients homozygous for the p.Arg1109X mutation in SH3TC2," Neuromuscular Disorders, vol. 16, no. 7, pp. 449-453, 2006.

[71] R. Claramunt, T. Sevilla, V. Lupo et al., "The p.R1109X mutation in SH3TC2 gene is predominant in Spanish Gypsies with Charcot-Marie-Tooth disease type 4," Clinical Genetics, vol. 71, no. 4, pp. 343-349, 2007.

[72] E. Arnaud, J. Zenker, A. S. de Preux Charles et al., "SH3TC2/KIAA1985 protein is required for proper myelination and the integrity of the node of Ranvier in the peripheral nervous system," Proceedings of the National Academy of Sciences of the United States of America, vol. 106, no. 41, pp. 17528-17533, 2009.

[73] V. Lupo, M. I. Galindo, D. Martínez-Rubio et al., "Missense mutations in the SH3TC2 protein causing CharcotMarie-Tooth disease type $4 \mathrm{C}$ affect its localization in the plasma membrane and endocytic pathway," Human Molecular Genetics, vol. 18, no. 23, pp. 4603-4614, 2009.

[74] R. C. Roberts, A. A. Peden, F. Buss et al., "Mistargeting of SH3TC2 away from the recycling endosome causes CharcotMarie-Tooth disease type 4C," Human Molecular Genetics, vol. 19, no. 6, pp. 1009-1018, 2009.

[75] C. Stendel, A. Roos, H. Kleine et al., "SH3TC2, a protein mutant in Charcot-Marie-Tooth neuropathy, links peripheral nerve myelination to endosomal recycling," Brain, vol. 133, no. 8, pp. 2462-2474, 2010.

[76] R. H. Baloh, "Mitochondrial dynamics and peripheral neuropathy," Neuroscientist, vol. 14, no. 1, pp. 12-18, 2008.

[77] Z. Sahenk, "Neurotrophins and peripheral neuropathies," Brain Pathology, vol. 16, no. 4, pp. 311-319, 2006.

[78] P. N. Hoffman and R. J. Lasek, "Axonal transport of the cytoskeleton in regenerating motor neurons: constancy and change," Brain Research, vol. 202, no. 2, pp. 317-333, 1980.

[79] I. G. McQuarrie and R. J. Lasek, "Transport of cytoskeletal elements from parent axons into regenerating daughter axons," Journal of Neuroscience, vol. 9, no. 2, pp. 436-446, 1989.

[80] P. Verma, S. Chierzi, A. M. Codd et al., "Axonal protein synthesis and degradation are necessary for efficient growth cone regeneration," Journal of Neuroscience, vol. 25, no. 2, pp. 331-342, 2005.

[81] D. E. Willis and J. L. Twiss, "The evolving roles of axonally synthesized proteins in regeneration," Current Opinion in Neurobiology, vol. 16, no. 1, pp. 111-118, 2006.

[82] X. Navarro, M. Vivó, and A. Valero-Cabré, "Neural plasticity after peripheral nerve injury and regeneration," Progress in Neurobiology, vol. 82, no. 4, pp. 163-201, 2007.

[83] J. Perea, A. Robertson, T. Tolmachova et al., "Induced myelination and demyelination in a conditional mouse model of Charcot-Marie-Tooth disease type 1A," Human Molecular Genetics, vol. 10, no. 10, pp. 1007-1018, 2001.

[84] E. Passage, J. C. Norreel, P. Noack-Fraissignes et al., "Ascorbic acid treatment corrects the phenotype of a mouse model of Charcot-Marie-Tooth disease," Nature Medicine, vol. 10, no. 4, pp. 396-401, 2004.

[85] F. Kaya, S. Belin, P. Bourgeois, J. Micaleff, O. Blin, and M. Fontés, "Ascorbic acid inhibits PMP22 expression by reducing cAMP levels," Neuromuscular Disorders, vol. 17, no. 3, pp. 248-253, 2007.

[86] D. Pareyson, M. M. Reilly, A. Schenone et al., "Ascorbic acid in Charcot-Marie-Tooth disease type 1A (CMT-TRIAAL and CMT-TRAUK): a double-blind randomised trial," The Lancet Neurology, vol. 10, no. 4, pp. 320-328, 2011.

[87] F. Désarnaud, A. N. Do Thi, A. M. Brown et al., "Progesterone stimulates the activity of the promoters of peripheral myelin protein-22 and protein zero genes in Schwann cells," Journal of Neurochemistry, vol. 71, no. 4, pp. 1765-1768, 1998.

[88] M. W. Sereda, G. Meyer zu Hörste, U. Suter, N. Uzma, and K. A. Nave, "Therapeutic administration of progesterone antagonist in a model of Charcot-Marie-Tooth disease (CMT-1A)," Nature Medicine, vol. 9, no. 12, pp. 1533-1537, 2003.

[89] G. M. zu Horste, T. Prukop, D. Liebetanz, W. Mobius, K. A. Nave, and M. W. Sereda, "Antiprogesterone therapy uncouples axonal loss from demyelination in a transgenic rat model of CMT1A neuropathy," Annals of Neurology, vol. 61, no. 1, pp. 61-72, 2007.

[90] M. Khajavi, K. Shiga, W. Wiszniewski et al., "Oral curcumin mitigates the clinical and neuropathologic phenotype of the Trembler-J mouse: a potential therapy for inherited neuropathy," American Journal of Human Genetics, vol. 81, no. 3, pp. 438-453, 2007.

[91] C. d'Ydewalle, J. Krishnan, D. M. Chiheb et al., "HDAC6 inhibitors reverse axonal loss in a mouse model of mutant HSPB1-induced Charcot-Marie-Tooth disease," Nature Medicine, vol. 17, no. 8, pp. 968-974, 2011.

[92] E. Soragni, C. Xu, A. Cooper, H. L. Plasterer, J. R. Rusche, and J. M. Gottesfeld, "Evaluation of histone deacetylase inhibitors as therapeutics for neurodegenerative diseases Methods," Molecular Biology, vol. 793, pp. 495-508, 2011.

[93] E. Verdú and X. Navarro, "The role of Schwann cell in nerve regeneration," in Understanding Glial Cells, B. Castellano, B. González, and M. Nieto Sampedro, Eds., pp. 319-359, Kluwer Academic, Boston, Mass, USA, 1998.

[94] L. E. Chen, K. Liu, A. V. Seaber, S. Katragadda, C. Kirk, and J. R. Urbaniak, "Recombinant human Glial growth factor 2 (rhGGF 2) improves functional recovery of crushed peripheral nerve (a double-blind study)," Neurochemistry International, vol. 33, no. 4, pp. 341-351, 1998.

[95] C. Birchmeier and K. A. Nave, "Neuregulin-1, a key axonal signal that drives Schwann cell growth and differentiation," Glia, vol. 56, no. 14, pp. 1491-1497, 2008.

[96] J. Newbern and C. Birchmeier, "Nrg1/ErbB signaling networks in Schwann cell development and myelination," Seminars in Cell and Developmental Biology, vol. 21, no. 9, pp. 922-928, 2010.

[97] Z. Sahenk, H. N. Nagaraja, B. S. McCracken et al., "NT-3 promotes nerve regeneration and sensory improvement in CMT1A mouse models and in patients," Neurology, vol. 65, no. 5, pp. 681-689, 2005. 
[98] M. Bibel and Y. A. Barde, "Neurotrophins: key regulators of cell fate and cell shape in the vertebrate nervous system," Genes and Development, vol. 14, no. 23, pp. 2919-2937, 2000.

[99] H. Funakoshi, M. Risling, T. Carlstedt et al., "Targeted expression of a multifunctional chimeric neurotrophin in the lesioned sciatic nerve accelerates regeneration of sensory and motor axons," Proceedings of the National Academy of Sciences of the United States of America, vol. 95, no. 9, pp. 5269-5274, 1998.

[100] K. Haastert and C. Grothe, "Gene therapy in peripheral nerve reconstruction approaches," Current Gene Therapy, vol. 7, no. 3, pp. 221-228, 2007.

[101] S. Zacchigna and M. Giacca, "Chapter 20 gene therapy perspectives for nerve repair," International Review of Neurobiology, vol. 87, pp. 381-392, 2009.

[102] J. Homs, L. Ariza, G. Pagès et al., "Schwann cell targeting via intrasciatic injection of AAV8 as gene therapy strategy for peripheral nerve regeneration," Gene Therapy, vol. 18, no. 6, pp. 622-630, 2011.

[103] R. Kirchmair, A. B. Tietz, E. Panagiotou et al., "Therapeutic angiogenesis inhibits or rescues chemotherapyinduced peripheral neuropathy: taxol- and thalidomideinduced injury of vasa nervorum is ameliorated by VEGF," Molecular Therapy, vol. 15, no. 1, pp. 69-75, 2007.

[104] J. Sanchez-Ramos, S. Song, F. Cardozo-Pelaez et al., "Adult bone marrow stromal cells differentiate into neural cells in vitro," Experimental Neurology, vol. 164, no. 2, pp. 247-256, 2000 .

[105] L. E. Kokai, J. P. Rubin, and K. G. Marra, "The potential of adipose-derived adult stem cells as a source of neuronal progenitor cells," Plastic and Reconstructive Surgery, vol. 116, no. 5, pp. 1453-1460, 2005.

[106] J. Caddick, P. J. Kingham, N. J. Gardiner, M. Wiberg, and G. Terenghi, "Phenotypic and functional characteristics of mesenchymal stem cells differentiated along a Schwann cell lineage," Glia, vol. 54, no. 8, pp. 840-849, 2006.

[107] M. Tohill, C. Mantovani, M. Wiberg, and G. Terenghi, "Rat bone marrow mesenchymal stem cells express Glial markers and stimulate nerve regeneration," Neuroscience Letters, vol. 362, no. 3, pp. 200-203, 2004.

[108] G. Keilhoff, A. Goihl, K. Langnäse, H. Fansa, and G. Wolf, "Transdifferentiation of mesenchymal stem cells into Schwann cell-like myelinating cells," European Journal of Cell Biology, vol. 85, no. 1, pp. 11-24, 2006.

[109] K. M. Safford, K. C. Hicok, S. D. Safford et al., "Neurogenic differentiation of murine and human adiposederived stromal cells," Biochemical and Biophysical Research Communications, vol. 294, no. 2, pp. 371-379, 2002.

[110] P. A. Zuk, M. Zhu, P. Ashjian et al., "Human adipose tissue is a source of multipotent stem cells," Molecular Biology of the Cell, vol. 13, no. 12, pp. 4279-4295, 2002.

[111] P. J. Kingham, D. F. Kalbermatten, D. Mahay, S. J. Armstrong, M. Wiberg, and G. Terenghi, "Adipose-derived stem cells differentiate into a Schwann cell phenotype and promote neurite outgrowth in vitro," Experimental Neurology, vol. 207, no. 2, pp. 267-274, 2007.

[112] C. Mantovani, D. Mahay, M. Kingham, G. Terenghi, S. G. Shawcross, and M. Wiberg, "Bone marrow- and adiposederived stem cells show expression of myelin mRNAs and proteins," Regenerative Medicine, vol. 5, no. 3, pp. 403-410, 2010.
[113] P. G. Di Summa, P. J. Kingham, W. Raffoul, M. Wiberg, G. Terenghi, and D. F. Kalbermatten, "Adipose-derived stem cells enhance peripheral nerve regeneration," Journal of Plastic, Reconstructive and Aesthetic Surgery, vol. 63, no. 9, pp. 1544-1552, 2010. 

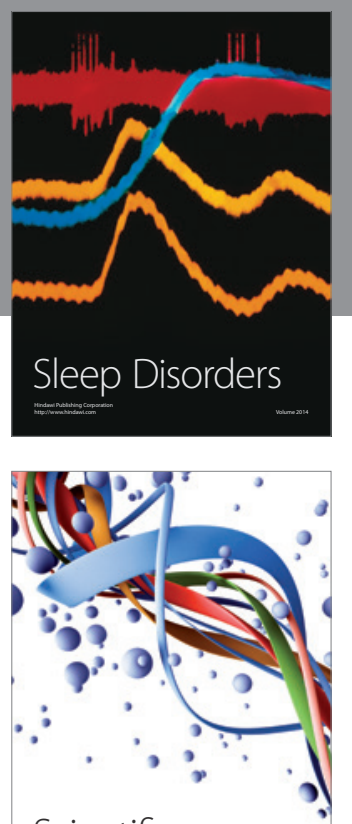

Scientifica
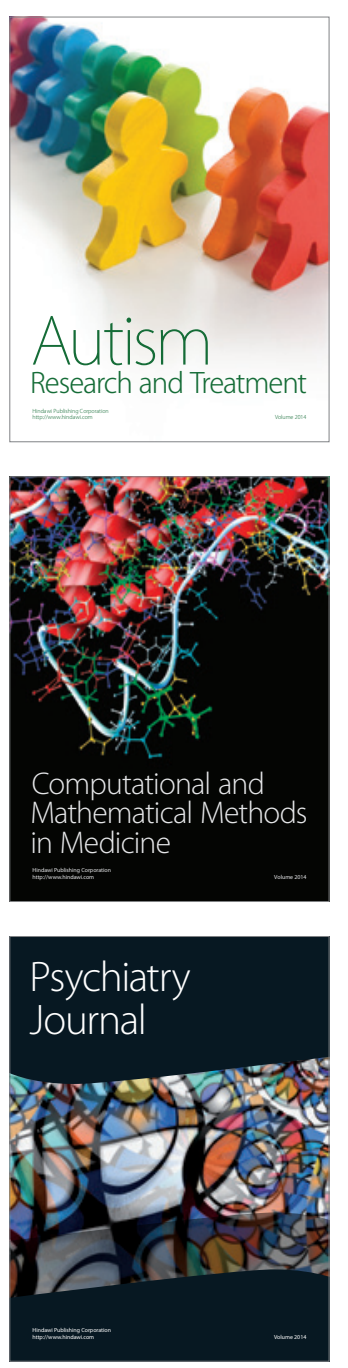
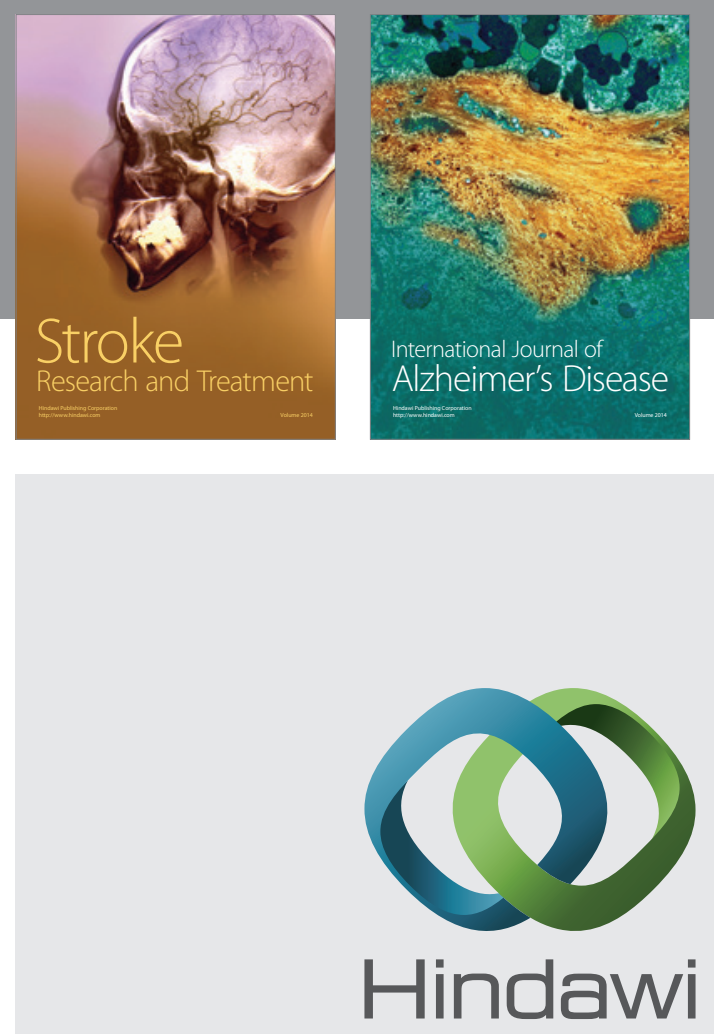

Submit your manuscripts at

http://www.hindawi.com
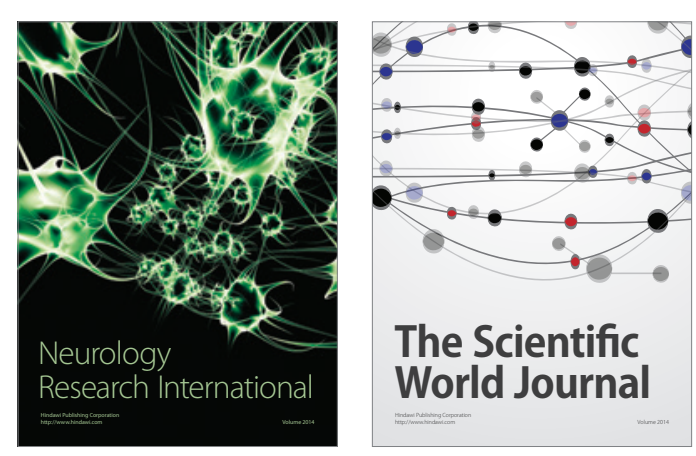

The Scientific World Journal

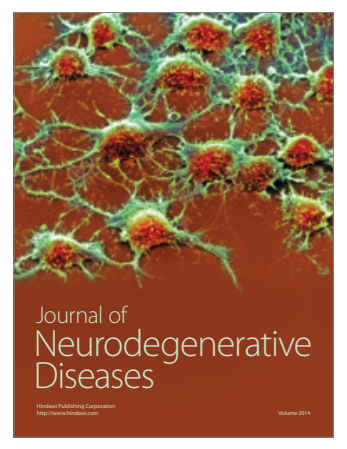

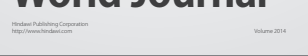

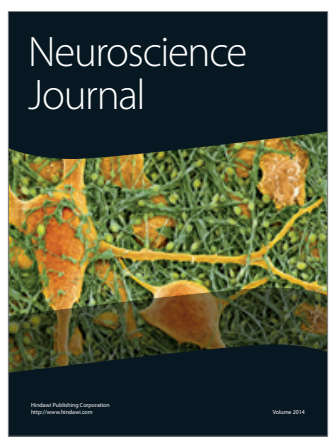

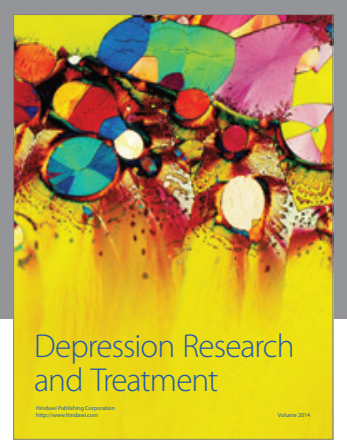
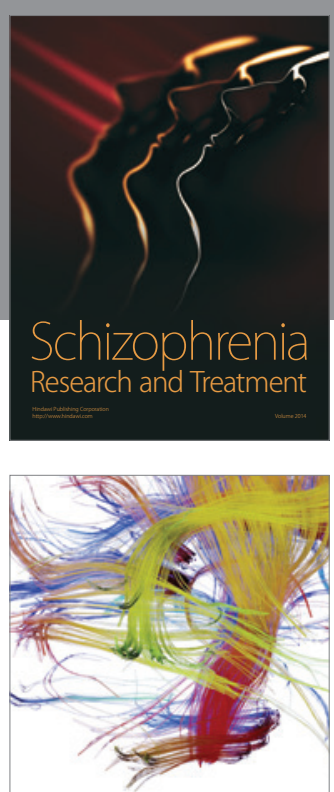

Brain Science

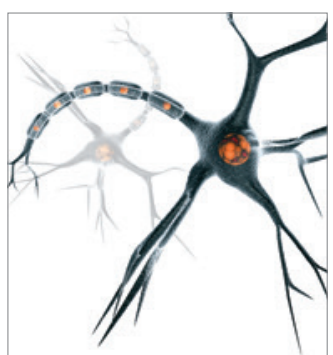

Neural Plasticity
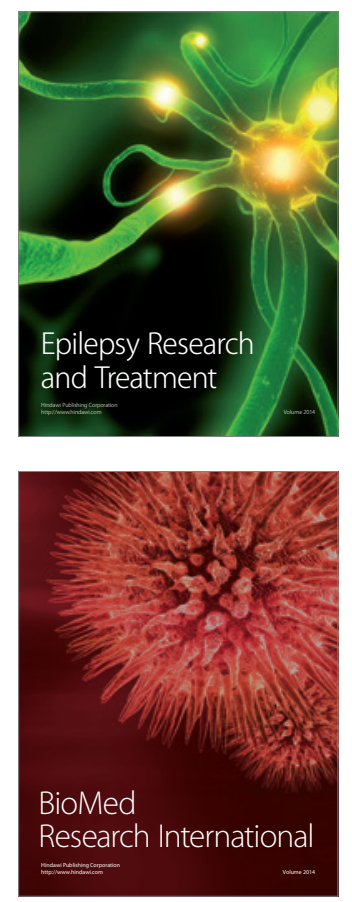

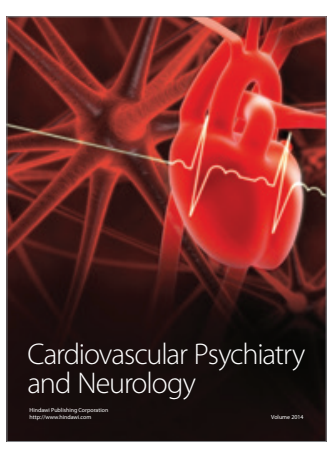

Parkinson's

Disease
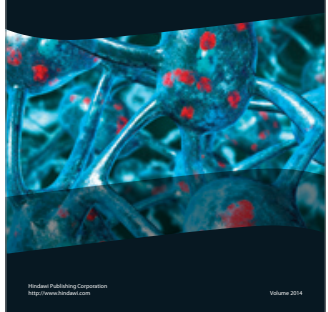